\title{
Diacronie
}

Studi di Storia Contemporanea

$N^{\circ} 9,1 \mid 2012$

Quando la classe operaia andava in paradiso

\section{Aida. Le Italie segrete dalla Resistenza a Tangentopoli}

\section{Steven Forti}

\section{Q OpenEdition \\ Journals}

\section{Edizione digitale}

URL: http://journals.openedition.org/diacronie/3116

DOI: $10.4000 /$ diacronie.3116

ISSN: 2038-0925

Editore

Association culturelle Diacronie

Notizia bibliografica digitale

Steven Forti, « Aida. Le Italie segrete dalla Resistenza a Tangentopoli », Diacronie [Online], № 9, 1 । 2012, documento 20, Messo online il 29 janvier 2012, consultato il 22 septembre 2020. URL : http:// journals.openedition.org/diacronie/3116; DOI : https://doi.org/10.4000/diacronie.3116 
N. 9 | 1|2012 Quando la classe operaia andava in paradiso

$\mathbf{2 0 /}$

\section{RECENSIONE:}

\section{Aida. Le Italie segrete dalla Resistenza a}

\section{Tangentopoli}

GOTOR, Miguel, Il memoriale della Repubblica : Gli scritti di Aldo Moro dalla prigionia e l'anatomia del potere italiano, Torino, Einaudi, 2011, 624 pp.

GIANNULI Aldo, Il Noto servizio, Giulio Andreotti e il caso Moro, Milano, Marco Tropea, 2011, 445 pp.

DE PROSPO Silvano, PRIORE Rosario, Chi manovrava le Brigate rosse? Storia e misteri dell'Hyperion di Parigi, scuola di lingue e centrale del terrorismo internazionale, Milano, Ponte alle Grazie, 2011, 306 pp.

A cura di Steven FORTI *

Nell'anno delle celebrazioni del 150 anniversario dell'Unità d'Italia non poteva mancare nel panorama bibliografico la presenza di opere dedicate a un argomento su cui tanto si è detto e tanto si è scritto: il caso Moro. Con i suoi vari corollari: le Brigate rosse, i servizi segreti, la strategia della tensione, gli anni di piombo, il che, in pratica, significa tentare di scrivere una parte cospicua della storia italiana tra la Resistenza e Tangentopoli.

Fare luce su questi aspetti non è stata cosa facile, in particolare negli ultimi tre decenni, per le difficoltà o l'impossibilità di consultare archivi e documenti, privati, ma soprattutto pubblici. Sul caso Moro sono state scritte decine e decine di pubblicazioni: memorie dei protagonisti e delle comparse, libri giornalistici contenenti inaspettate rivelazioni (spesso scandalistiche e sovente infondate) e i più disparati studi in ambito storico, a sostegno delle tesi dei cosiddetti dietrologi così come dei cosiddetti avantologi o negazionisti ${ }^{1}$. Come ha notato Miguel Gotor, sul caso Moro esiste una

\footnotetext{
${ }_{1}^{1}$ A titolo d'esempio, vedasi per quanto riguarda le memorie e le interviste a ex brigatisti: FRANCESCHINI, Alberto, BUFFA, Pier Vittorio, GIUSTOLISI, Franco, Mara Renato e io: Storia dei fondatori delle BR, Milano, Mondadori, 1988; CURCIO, Renato, A viso aperto, Milano, Mondadori, 1993; MORETTI, Mario, Brigate Rosse: Una storia italiana, Milano, Anabasi, 1994; BRAGHETTI, Anna Laura, Il prigioniero, Milano, Mondadori, 1998;
} 
sovrabbondanza interpretativa a fronte dell'assenza di un lavoro storico su carte e documenti. Lo stesso disorso può essere fatto relativamente agli altri corollari di cui più sopra si diceva. Le ragioni e le responsabilità sono molte e in parte ce le aveva spiegate qualche anno fa proprio un altro degli autori di cui stiamo trattando, Aldo Giannuli, in una sorta di j’accuse allo Stato italiano che cercava di portare l'attenzione dell'opinione pubblica sullo stato di conservazione e di consultazione degli archivi di Stato². Qualche passo in avanti è stato fatto in questo 2011 - in gran parte grazie agli ottimi studi di Gotor e Giannuli - ma c'è ancora molto da lavorare, molto da scoprire, molto da sapere. Come scrive Giannuli, «Diversi passaggi ci sfuggono ancora, perché troppi documenti sono inaccessibili e troppe bocche cucite. Possiamo solo supporne alcuni»3. Andiamo però con ordine e vediamo di cosa trattano questi tre corposi libri, usciti a breve distanza di tempo, tra la primavera e l'autunno dell'anno appena concluso.

Ne Il memoriale della Repubblica, Miguel Gotor affronta un nodo gordiano della vicenda Moro, ricostruendo le peripezie del cosiddetto memoriale, scritto dallo statista democristiano durante i 55 giorni di prigionia, proponendo un'attenta analisi filologica del testo4. L'indagine di Gotor segue le complesse vicissitudini di questo straordinario documento, dalla sua parziale diffusione e pubblicazione ancora durante il sequestro di Moro - 8 pagine autografe riguardanti il dirigente democristiano Paolo Emilio Taviani diffuse il 9 aprile 1978 come allegato al comunicato numero 5 delle $\mathrm{Br}$ - fino al ritrovamento della versione apparentemente completa del memoriale -421 fotocopie dei dattiloscritti originali - nell'intercapedine del covo delle $\mathrm{Br}$ di via Monte Nevoso a

GALLINARI, Prospero, Un contadino nella metropoli: Ricordi di un militante delle Brigate Rosse, Milano, Bompiani, 2006. Per quanto riguarda gli studi storici si rimanda a FLAMIGNI, Sergio, La tela del Ragno : Il delitto Moro, Milano, Kaos, 1993; ID., Convergenze parallele: Le Brigate rosse, i servizi segreti e il delitto Moro, Milano, Kaos, 1998; BISCIONE, Francesco Maria, Il delitto Moro, Roma, Editori Riuniti, 1998; ID., Il sommerso della Repubblica: La democrazia italiana e la crisi dell'antifascismo, Torino, Bollati Boringhieri, 2003; GIOVAGNOLI, Agostino, Il caso Moro: una tragedia repubblicana, Bologna, Il Mulino, 2005; SATTA, Vladimiro, Il Caso Moro ed i suoi falsi misteri, Soveria Mannelli, Rubbettino, 2006; DE LUTIIS, Giuseppe, Il golpe di via Fani, Milano, Sperling \& Kupfer, 2007; GRASSI, Stefano, Il caso Moro: Un dizionario italiano, Milano, Mondadori, 2008. Non entriamo qui per ovvie ragioni di spazio nell'ambito delle pubblicazioni giornalistiche né in quello delle memorie o delle interviste ad altri dirigenti politici, membri delle forze dell'ordine, familiari e giornalisti.

${ }^{2}$ GIANNULI, Aldo, L'armadio della Repubblica, Roma, Nuova Iniziativa Editoriale, 2005.

3 GIANNULI, Aldo, Il Noto servizio, Giulio Andreotti e il caso Moro, Milano, Marco Tropea, 2011, p. 326.

4 GOTOR, Miguel, Il memoriale della Repubblica: Gli scritti di Aldo Moro dalla prigionia e l'anatomia del potere italiano, Torino, Einaudi, 2011. Vedasi anche la recensione di BREDA, Marzio, «I segreti d'Italia nelle parole di Moro», Il Corriere della Sera, 4 maggio 2011; consultabile anche on-line,

URL: < http://www.corriere.it/cultura/11_maggio_04/segreti-italia-moro-breda_96f8bogc7611-11eo-b432-72ecee218af7.shtml > [consultato il 15 gennaio 2012]. Per ulteriori materiali relativi a questo volume - interviste radiofoniche e televisive, registrazioni di interventi in seminari e giornate di studio, ecc. - vedasi il blog di Miguel Gotor,

URL: < http://miguelgotor.italianieuropei.it/ > [consultato il 15 gennaio 2012]. 
Milano il 10 ottobre 1990, passando per il precedente ritrovamento del memoriale nello stesso appartamento il primo ottobre del 1978 e la sua parziale pubblicazione - solo 49 pagine dattiloscritte - il successivo 17 ottobre, su indicazione del Presidente del Consiglio Andreotti a seguito di una fuga di notizie.

Tutto ciò viene inquadrato nel contesto politico italiano ed internazionale della seconda metà del Novecento: questo per via della stretta relazione che unisce il memoriale di Moro e la sua diffusione parziale - o, sarebbe meglio dire, la sua censura - con molti dei momenti chiave della storia dell'Italia repubblicana, dal caso Pecorelli al caso Dalla Chiesa - le cui morti ebbero molto probabilmente relazione diretta con le ricerche del memoriale Moro - dalle responsabilità del golpe Borghese e della strategia della tensione alle attività della Nato e della Cia, dal caso Solo5 alla Loggia massonica P2, dalla figura di Sindona a Gladio, dalle clausole del cosiddetto lodo Moro sul conflitto israelo-palestinese in Italia alla fuga del criminale nazista Kappler, fino alla questione delle tangenti Italcasse e Lockheed.

Gotor ci spiega che proprio nell'ambito di tale contesto sta la soluzione del caso Moro $^{6}$. Con estremo rigore storico e grazie ad un apparato bibliografico ricchissimo, composto da tutta la bibliografia secondaria e la memorialistica esistente sull'argomento, a partire da un quasi completo spoglio della stampa quotidiana e periodica e dalla consultazione di numerosi documenti d'archivio - soprattutto, quelli dei cinque processi e delle due commissioni parlamentari sul caso Moro -, Gotor spiega come la versione del memoriale trovata nell'ottobre del 1990 dimostri che vi fu una censura sul memoriale divulgato nell'ottobre del 1978. Secondo Gotor, vi furono due mani censorie - probabilmente quelle del gruppo antiterrorismo di Dalla Chiesa e quelle di qualche agente del servizio segreto italiano - che tra l'1 e il 17 ottobre 1978 agirono sui dattiloscritti e sui manoscritti trovati in via Monte Nevoso. La tesi sostenuta da Gotor dimostrerebbe che, da un certo momento in poi, il memoriale non fosse più in mano alle Brigate rosse, differendo in questo dall'opinione di Giannuli. Il punto chiave è, tuttavia, un altro: la probabile, ma solo supposta fino ad ora, esistenza di un ur-

\footnotetext{
5 Sulla questione si rinvia al recente studio di FRANZINELLI, Mimmo, Il piano Solo: I servizi segreti, il centro-sinistra e il «golpe» del 1964, Milano, Mondadori, 2010, di cui proprio Gotor scrisse una positiva recensione. Vedasi, GOTOR, Miguel, «Su quel golpe il veto Usa - Il Piano Solo", Il Sole 24 ore, 28 novembre 2010. Consultabile anche on-line URL: < http://miguelgotor.italianieuropei.it/ > [consultato il 15 gennaio 2012].

6 Già nel 2009, Giambattista Scirè notava che «anche la maniera in cui la storiografia ha affrontato, salvo rari casi, la vicenda, senza riuscire a fornirne una credibile interpretazione politica complessiva che tenga conto del macronodo relativo ai rapporti tra la dimensione nazionale e internazionale, è emblematica della difficoltà nell'affrontare la lunga crisi degli anni Settanta e i veri motivi della fine della Prima Repubblica», in SCIRÈ, Giambattista, «Il caso Moro: Frammenti di una verità indicibile», in Italia Contemporanea, 255, 2009, p. 276.
} 
memoriale, che sarebbe dimostrata da alcuni "lettori precoci", come, tra gli altri, Pecorelli e Dalla Chiesa. Tale ur-memoriale occulterebbe secondo Gotor tre questioni di capitale importanza per gli equilibri politici italiani della Prima Repubblica: il golpe Borghese, il cosiddetto lodo Moro e la fuga di Kappler. Se le parti del manoscritto rese note nell'ottobre 1990 - è solo casuale la coincidenza temporale del secondo ritrovamento in via Monte Nevoso con la dichiarazione della pubblica esistenza di Gladio da parte dell'allora presidente del Consiglio Andreotti? - non erano ormai più pericolose in quel momento politico nazionale ed internazionale, le tre questioni cui si accennava prima lo erano ancora per implicazioni di carattere giudiziario e per la ragion di Stato ${ }^{7}$. A questo si deve, secondo Gotor, il fatto che non disponiamo ancora oggi del memoriale di Moro nella sua integrità.

Miguel Gotor, ricercatore di storia moderna presso l’Università degli Studi di Torino e collaboratore de «Il Sole 24 ore» e de «la Repubblica», non è nuovo a questo tipo di studi, avendo curato recentemente un'edizione delle lettere scritte dalla prigionia dallo stesso $\mathrm{Moro}^{8}$. Nell'attenzione prestata al documento si nota la sua formazione di storico modernista, esperto di Inquisizione, eretici e Controriforma nel Cinquecento italiano. Un'attenzione che offre una nuova prospettiva d'analisi, allontanandosi dalle troppo frequenti ricostruzioni giornalistiche, dove la dietrologia la fa da padrona, e proponendo un'interessante e innovatrice indagine sui meccanismi di funzionamento del potere italiano sul lungo periodo. Il potere, ben inteso, come qualcosa di diverso dalla politica. ${ }^{9}$ Per fare ciò, Gotor stabilisce una fertile relazione tra il Moro libero - un Moro che pensa come uomo politico - ed il Moro prigioniero - un Moro che seppure in cattività, continua a ragionare e pensare come uomo di Stato - che gli serve per poter giungere ad un'interpretazione di un Moro «prigioniero liberato». Sarebbe a dire, un Moro finalmente libero dalla prigionia creata dalle lettere e dal memoriale scritti durante i cinquantacinque giorni grazie alla liberazione del messaggio in essi contenuto. Critico con la maggior parte dei giudizi dati da esponenti politici, giornalisti e parte degli studiosi riguardo alle parole di Moro - come ad esempio, quelli

\footnotetext{
7 Infatti, il processo sul lodo Moro si chiuse solamente nel 1991, mentre la fuga di Kappler fu organizzata dal cosiddetto Noto servizio, la cui esistenza non è mai stata confermata ufficialmente e di cui non si voleva si sapesse nulla, meno che mai con la DC saldamente al governo, come conferma anche Giannuli.

8 MORO, Aldo, Le lettere dalla prigionia, a cura di GOTOR, Miguel, Torino, Einaudi, 2008.

9 Una distinzione - quella tra potere e politica - su cui qualche anno fa ha scritto delle belle pagine Valerio Romitelli Cfr. ROMITELLI, Valerio, Storie di politica e di potere, Napoli, Cronopio, 2004. L'idea del potere - in questo caso italiano - è ben rappresentata dalla copertina del libro di Gotor pubblicato da Einaudi con il ritratto di Antonello da Messina, con uno sguardo "enigmatico, ambiguo e indecifrabile", come ha spiegato lo stesso Gotor.
} 
che consideravano incomprensibile il suo linguaggio, tra cui Leonardo Sciascia ${ }^{10}$ Gotor riafferma la grande lucidità politica dello statista democristiano, che ha saputo anticipare molti dei fatti avvenuti nel decennio successivo ${ }^{11}$.

La tesi di Gotor è che Moro fu una specie di barometro e frangiflutti della politica democristiana (ed italiana in genere), capace di mantenere entro la legalità democratica un paese di frontiera come era l'Italia negli anni della Guerra fredda. Centrale diviene dunque la strategia di Moro, fautore di una politica di apertura al centro sinistra sia nei primi anni Sessanta con il PSI di Nenni sia a metà anni Settanta con il PCI di Berlinguer, in contrasto con le tendenze conservatrici di gran parte della Democrazia cristiana. La morte di Moro viene a significare dunque, per Gotor, la fine della “involuzione moderata della Dc", con la vittoria dell'ala andreottiana e la corsa al baratro degli anni Ottanta.

Le informazioni e le interpretazioni contenute ne Il memoriale della Repubblica una lettura imprescindibile per chiunque voglia affrontare lo studio del caso Moro e dell'Italia dei decenni Sessanta e Settanta negli anni a venire - si integrano con quelle contenute in un altro volume che si occupa di ampliare la prospettiva d'analisi, inserendo la realtà italiana nelle dinamiche internazionali, senza per questo scadere in futili ed autoreferenziali spy story. Stiamo parlando de Il Noto servizio, Giulio Andreotti e il caso Moro, l'ultima fatica di Aldo Giannuli, che da oltre un quindicennio lavora sulla storia dell'Italia repubblicana ${ }^{12}$. Già consulente delle Commissioni parlamentari sulle stragi e sul caso Mitrokhin e delle procure della Repubblica di Brescia, Milano, Palermo e Roma, oltre che dell'Ufficio istruzione del tribunale di Milano, lo storico dell'Università statale di Milano presenta un lavoro davvero eccezionale per la quantità e la qualità dei documenti d'archivio consultati ${ }^{13}$. Grazie ad

${ }^{10}$ SCIASCIA, Leonardo, L'affaire Moro, Palermo, Sellerio, 1978.

${ }^{11}$ All'evidente recupero della figura politica ed intellettuale di Moro l'autore affianca una critica, per nulla velata, della sinistra extraparlamentare italiana - soprattutto il mondo dell'Autonomia - con alcune considerazioni che, ci sembra, scadono in alcuni casi in un giudizio ex post non esente da un certo moralismo.

${ }_{12}$ GIANNULI Aldo, Il Noto servizio, Giulio Andreotti e il caso Moro, Milano, Marco Tropea, 2011. Si vedano anche le recensioni di STAJANO, Corrado, «Il pozzo nero della Repubblica», in Il Corriere della Sera, 24 ottobre 2011, consultabile anche on-line all'URL: < http://www.corriere.it/unita-italia-15o/recensioni/11_ottobre_24/giannuli-il-noto-

servizio_eo68abee-fe4f-11eo-bb8b-fd7e32debc75.shtml > [consultato il 15 gennaio 2012] e di LUNARDINI, Matteo, “"Il Noto servizio, Giulio Andreotti e il caso Moro": Aldo Giannuli riscrive la 'mala Italia'», in Il Fatto Quotidiano, 9 novembre 2011, consultabile anche on-line all'URL: < http://www.ilfattoquotidiano.it/2011/11/o9/noto-servizio-giulio-andreotti-caso-moro-aldogiannuli-riscrive-mala-italia/169565/ > [consultato il 15 dicembre 2012].

${ }_{13}$ Oltre a documenti conservati in archivi pubblici quali l'Archivio centrale dello Stato ed altri istituti e fondazioni, gli archivi consultati sono stati 63. Le fonti provengono dal "famoso" deposito della via Appia - scoperto dallo stesso Giannuli nel 1996 con moltissimi documenti del Ministero degli Interni, soprattutto dell'Ufficio affari riservati dell'Archivio della direzione 
una buona contestualizzazione storica, Giannuli riscrive la storia d'Italia dall'armistizio dell'8 settembre 1943 agli ultimi mesi della Prima Repubblica, seguendo le vicende del cosiddetto Noto servizio dai suoi esordi alla sua fine. Il suo libro è una vera e propria miniera di informazioni, capace di sbrogliare una matassa enorme di documenti e di seguire un'infinità di piste, ricostruendo le biografie di decine e decine di personaggi, finora poco conosciuti, e di metterne in luce relazioni e legami ${ }^{14}$.

Quella del Noto servizio è una storia complessa, spesso indecifrabile, caratterizzata principalmente dall'assenza di una denominazione precisa di questa sorta di servizio parallelo, che è sempre rimasto nell'ombra e che si è sempre voluto tenere segreto, ancora più di Gladio. Come spiega Giannuli, il Noto servizio «non era mai stato istituito formalmente, era un organismo estremamente fluido, privo di organigrammi e bilanci», «una sorta di "funzione esterna" del servizio militare», che poteva cambiare pelle e componenti nel tempo, ma la cui caratteristica era che «il suo ambito d'azione era costantemente circoscritto in un triangolo i cui vertici erano l'Arma dei carabinieri e il servizio segreto militare, poi gli ambienti imprenditoriali prossimi alla Confindustria e, infine, i servizi segreti americani», oltre al referente politico che è sempre stato Giulio Andreotti, definito più esattamente «l'interfaccia politica di questa struttura a metà strada fra militari e imprenditori» ${ }^{15}$.

Il Noto servizio era dunque una «struttura che andava bene per i "dirty jobs"», il prodotto di «una sorta di eterogenesi dei fini» tra la Cia, gli ambienti imprenditoriali, i militari e i carabinieri, la cui funzione fu sempre «l'azione anticomunista, filoatlantica e filoimprenditoriale». Insomma, «un vero e proprio scheletro nell'armadio della repubblica». A Giannuli preme sottolineare anche «l'idea di un ruolo diretto e autonomo degli ambienti imprenditoriali, ben al di là del semplice finanziamento», il

centrale della polizia di prevenzione - e dalle istruttorie penali "Salvini" (confluita nei dibattimenti per la strage di Piazza Fontana e di via Fatebenefratelli), "Mastelloni" (riguardante il caso "Argo 16"), "Ingroia" (sul caso De Mauro), "Piantoni-De Martino" (sulla strage di Brescia), "Casson" (sul caso Gladio) e dall'istruttoria penale sul caso Italicus. Inoltre, sono stati consultati gli archivi delle commissioni parlamentari di inchiesta sul caso Moro, sul caso EniPetromin, sulla loggia massonica P2, sulle stragi e sul caso Mitrokhin. Vedasi, GIANNULI, Aldo, Il Noto servizio, cit., p. 23-25.

${ }_{14} \mathrm{Il}$ fatto di riuscire a riassumere questa immensa quantità di informazioni in sole 400 pagine è un grande merito dell'autore. E proprio per questo non ci si spiega come si sia deciso di non includere un indice dei nomi, che risulterebbe di estrema utilità per ricostruire in un secondo momento alcune di queste traiettorie biografiche.

15 L'assenza di un nome vero e proprio fu favorita «dalla particolarissima situazione di irregolarità amministrativa della Presidenza del Consiglio, almeno nella sua prima fase, per il tramite dell'Ufficio zone di confine», in GIANNULI, Aldo, Il Noto servizio, cit., pp. 15, 16, 18, 386. Senza cadere negli sterili dietrologismi dell'Andreotti grande burattinaio della Repubblica, Giannuli considera più realisticamente che il Noto servizio fu «uno degli strumenti della sua azione politica» GIANNULI, Aldo, Il Noto servizio, cit., p. 391. 
che porterebbe a riconsiderare «l'intera vicenda della strategia della tensione [...] alla luce di un altro aspetto che l'ha attraversata: quella del conflitto di classe» ${ }^{16}$.

Il punto di partenza è il rapporto di Alberto Grisolia, un confidente del nucleo milanese dell'Ufficio affari riservati, datato 4 aprile 1972, che sembra far luce, anche se solo parzialmente, su questo finora sconosciuto servizio segreto parallelo ${ }^{17}$. Dal lungo rapporto di Grisolia, Giannuli ricostruisce la lunga vita del Noto servizio nelle sue varie fasi, a partire dai primissimi mesi successivi alla Liberazione con l'analisi della figura chiave del generale Mario Roatta, di importanti settori del clandestinismo fascista ${ }^{18}$, della Resistenza bianca di Edgardo Sogno e di una creazione di quest'ultimo, l'associazione Pace e Libertà, nata nel 1954 e nella quale troviamo un altro controverso personaggio, l'ex comunista Luigi Cavallo. Nei primi anni Sessanta, il filo rosso del Noto servizio è nuovamente visibile, intervenendo prima a favore, poi contro il governo di centro sinistra, attraverso figure quali Felice Fulchignoni, Carlo Fumagalli e Sigfrido Battaini, e dimostrando strettissimi vincoli con l'estrema destra di Ordine Nuovo di Pino Rauti ${ }^{19}$.

La strage di piazza Fontana del 12 dicembre 1969 aprì una nuova fase nella quale il Noto servizio fu tristemente protagonista. Giannuli dimostra le responsabilità - o, almeno, la non estraneità - di questo servizio parallelo nel fallito golpe Borghese, nelle morti di Feltrinelli e di Calabresi - probabilmente dovute alla conoscenza di uno scambio armi/petrolio tra il governo italiano e la Libia di Gheddafi - nel caso Montedison, negli scandali legati a figure di primo piano del PSI $^{20}$ e nella strage di via

${ }^{16}$ GIANNULI, Aldo, Il Noto servizio, cit., pp. 387, 388, 392.

$17 \mathrm{Il}$ documento è riprodotto interamente in appendice al testo, insieme ad altri due documenti invero assai interessanti: la nota "All'insegna della Trama nera", sempre del 1972, e il cosiddetto Testamento Borghese del 1974. Ad essi l'autore affianca "Diciotto domande a Mario Moretti e agli altri ex dirigenti delle Br" sui coni d'ombra della vicenda Moro, in GIANNULI, Aldo., Il Noto servizio, cit., pp. 403-417.

${ }_{18}$ Vedasi nello specifico anche, GIANNULI, Aldo, «Le spie di Salò: Doppi giochi e tanti soldi per salvare la pelle e infiltrare la democrazia», L'Unità, 25 aprile 2009, consultabile anche in www.aldogiannuli.it, dove si possono trovare altri materiali relativi a questo volume e alle altre pubblicazioni e ricerche dell'autore.

19 GIANNULI, Aldo, Il Noto servizio, cit., pp. 81-129. Secondo Giannuli, alla fine degli anni Cinquanta il Noto servizio si attivò per consentire al partito di Nenni di «disporsi su posizioni di netto anticomunismo». L'obiettivo era chiaro: isolare ed indebolire il Pci ed addomesticare il Psi, includendolo nella sfera democristiana. Nel giro di pochi anni, dopo le prime misure del governo di centro sinistra presieduto da Aldo Moro, la posizione del Noto servizio cambiò radicalmente: la forte opposizione della Confindustria alle politiche messe in atto dal governo come, la nazionalizzazione dell'energia elettrica - diede il via a una serie di azioni, in collaborazione con il Sifar e l'estrema destra, per destabilizzare l'alleanza Dc-Psi, tra le quali non è escluso il "piano Solo".

${ }^{20}$ Giannuli fa riferimento alle vicende che coinvolsero Giacomo Mancini, segretario del Partito socialista tra l'aprile del 1970 e il novembre del 1972, come il caso Anas e il caso delle intercettazioni telefoniche. Entrambi i casi furono sollevati dalla stampa di destra - soprattutto il «Candido» di Pisanò - in funzione antimanciniana: si considerava difatti che il Psi di Mancini 
Fatebenefratelli. Ma il Noto servizio non sparì dopo il rapporto di Grisolia: come era già accaduto, venne smantellato e poi ricostituito, con la costante presenza di alcune figure, quali il fantomatico padre Enrico Zucca, Giorgio Pisanò e Adalberto Titta. Tanto che il Noto servizio instaurò uno stretto legame con l'estrema destra, il traffico d'armi e la malavita organizzata e non fu del tutto estraneo ad altri oscuri episodi della storia di quegli anni, come il caso della Rosa dei Venti o le stragi di piazza della Loggia a Brescia e dell'Italicus o, ancora, l'evasione del criminale nazista Kappler nell'agosto del 1977.

Il protagonismo del Noto servizio continuò anche in seguito, toccando probabilmente il suo climax con il caso Moro. Qui risulta interessante vedere in dettaglio qual è la lettura offerta da Giannuli, che dedica un centinaio di pagine a questo punto. Insofferente all'ormai classica lettura dell'eliminazione di Moro come piano prestabilito delle $\mathrm{Br}$ per impedire l’ingresso comunista nel governo ed escludendo che «i brigatisti fossero il terminale consapevole di un servizio segreto americano» ${ }^{21}$, Giannuli avvalla la teoria del "doppio ostaggio" - la persona di Moro e le carte di Moro - elaborata da Giovanni Pellegrino, presidente della Commissione stragi tra il 1996 e il 2001, e conferisce un ruolo centrale all'esperto del dipartimento di Stato americano Steve Pieczenik, giunto a Roma alla fine di marzo del 1978, coadiuvato dal Noto servizio, in particolare nella persona di Adalberto Titta. Secondo Giannuli, il punto chiave è la volontà di tenere celati all'opinione pubblica, non tanto la questione di Gladio e di altri segreti relativi alla sicurezza nazionale - come spesso si è scritto - ma i «molti segreti della Dc e dei suoi principali esponenti, gli aspetti scabrosi della strategia della tensione ed episodi di corruzione politica», in quanto «le due trattative erano incompatibili tra loro, per cui la vera alternativa era o Moro vivo con le sue rivelazioni fuori controllo o le sue rivelazioni sotto controllo e Moro morto»22, l'obiettivo della "missione" di Pieczenik fu chiaramente la morte di Moro - dunque, far fallire il tentativo del partito della trattativa, rappresentato essenzialmente da Craxi - e il recupero del memoriale, rendendo innocue le Br. Insomma, come ha scritto Giambattista Scirè, quella di Moro fu la «cronaca di una morte annunciata» ${ }^{23}$. Un

potesse avere «un'elevata potenzialità espansiva sia sul fianco sinistro che su quello destro. Era quindi necessario normalizzare il Psi facendo cadere Mancini». Malgrado la controffensiva di Mancini che riuscì a bloccare queste iniziative, ricollegabili secondo Giannuli, al Noto servizio, nel XXXIX congresso del Psi del novembre 1972 Mancini perse la segreteria del partito, che fu occupata da De Martino. Vedasi, GIANNULI, Aldo, Il Noto servizio, cit., pp. 213-217.

${ }^{21}$ GIANNULI, Aldo, Il Noto servizio, cit., p. 296.

${ }^{22}$ GIANNULI, Aldo, Il Noto servizio, cit., pp. 320-321, 323. Per l'analisi di Giannuli, centrali risultano le rivelazioni rilasciate recentemente dallo stesso Pieczenik in AMARA, Emmanuel, Abbiamo ucciso Aldo Moro: Dopo 30 anni un protagonista esce dall'ombra, Roma, Cooper, 2008.

${ }^{23}$ SCIRÈ, Giambattista, op .cit., p. 276. 
obiettivo riuscito appieno, senza il bisogno di alcun infiltrato di lusso per realizzare un'eterodirezione del gruppo brigatista: «è stato sufficiente comprendere le deficienze culturali ed intellettuali del gruppo dirigente per portare le cose al punto desiderato» ${ }^{24}$. L'analisi di Giannuli distingue dunque tra un primo caso Moro - consistente nel rapimento, nella prigionia e nell'uccisione dello statista democristiano - ed un secondo caso Moro - consistente nelle sue rivelazioni - in cui si tirano in ballo la malavita e le figure di Pecorelli e Dalla Chiesa. Concordando sostanzialmente con l'analisi del memoriale di Moro datane da Gotor, Giannuli aggiunge però che il cosiddetto urmemoriale è «sempre rimasto in possesso di pochi dirigenti delle $\mathrm{Br}[\ldots]$ che hanno provveduto a occultarlo, probabilmente all'estero, e ha costituito la loro assicurazione sulla vita nel caso di una nuova Stammheim».

La fine del Noto servizio avverrebbe, secondo l'autore, nel periodo successivo al sequestro Cirillo, in una non troppo strana coincidenza temporale con la P2 e con una serie di morti sospette - ben 36 - di persone che ebbero rapporto o con il caso Moro o con il caso Cirillo o con entrambi. Il caso del sequestro dell'esponente della Dc campana Ciro Cirillo viene definito «in tutto e per tutto una replica in tono minore del caso Moro» 25 - fino addirittura al coinvolgimento della malavita e al ruolo chiave giocato dall'onnipresente Adalberto Titta - ma con un finale diverso - la liberazione dell'ostaggio e il mantenimento del segreto sulle rivelazioni fatte da Cirillo alle $\mathrm{Br}$.

Al caso Moro e all'esistenza di supposti servizi segreti e/o paralleli si lega direttamente anche il terzo libro di cui parliamo in queste pagine. Frutto del lavoro e delle ricerche del giornalista Silvano De Prospo e del magistrato romano Rosario Priore - che ha seguito alcuni dei più importanti casi della storia giudiziaria italiana, come Ustica e l'attentato contro Giovanni Paolo II - Chi manovrava le Brigate rosse? è un volume molto più agile dei precedenti, ma, a differenza di questi, è un testo non imprescindibile ${ }^{26}$. La prima metà del libro (pp. 27-135) è una breve storia delle Br dalle origini fino al caso Moro, che si basa essenzialmente sui lavori di Flamigni e di De Lutiis e sulle testimonianze di Franceschini, Moretti, Curcio e Morucci ${ }^{27}$. La seconda parte del libro (pp. 137-259), invece, è una storia del Superclan e della scuola di lingue

24 GIANNULI, Aldo, Il Noto servizio, cit., p. 301.

25 GIANNULI, Aldo, Il Noto servizio, cit., p. 354, 369.

${ }^{26}$ DE PROSPO Silvano, PRIORE Rosario, Chi manovrava le Brigate rosse? Storia e misteri dell'Hyperion di Parigi, scuola di lingue e centrale del terrorismo internazionale, Milano, Ponte alle Grazie, 2011.

${ }_{27}$ CURCIO, Renato, A viso aperto, cit.; MORETTI, Mario, Brigate Rosse: Una storia italiana, cit.; FLAMIGNI, Sergio, La sfinge delle Brigate Rosse, Milano, Kaos, 2004; FASANELLA, Giovanni, FRANCESCHINI, Alberto, Che cosa sono le BR, Milano, Rizzoli, 2004; MORUCCI, Valerio, La peggio gioventù: una vita nella lotta armata, Milano, Rizzoli, 2004; DE LUTIIS, Giuseppe, Il golpe di via Fani, cit. 
Hyperion di Parigi, basata sostanzialmente sulle indagini compiute dalla magistratura italiana nella prima parte degli anni Ottanta.

Tralasciando la prima parte, che non apporta nulla di nuovo agli studi esistenti sulla questione, vale la pena soffermarsi sulla seconda parte del libro. Secondo quanto esposto dagli autori, il Superclan - su cui invero poche certezze ci sono al giorno d'oggi - fu il gruppo facente capo a Corrado Simioni, Duccio Berio e Vanni Mulinaris, che fu molto vicino a Renato Curcio e Mara Cagol nel periodo trentino e nel biennio milanese 1969-1970, un biennio segnato dall'esperienza del Collettivo politico metropolitano e di Sinistra proletaria. La rottura tra il gruppo di Simioni e le future Br ebbe luogo tra la primavera e l'estate del 1970, tra l'incontro di Chiavari e quello di Pecorile, presumibilmente a causa di due concezioni distinte dell'organizzazione e della lotta politica, contraria alla clandestinità e più legata alla realtà delle fabbriche quella delle $\mathrm{Br}$ dei primi tempi, favorevole alla clandestinità e ad azioni sanguinarie contro obiettivi americani ed israeliani quella del Superclan.

Secondo gli autori, il Superclan fu un gruppo «strutturato e organizzato in cellule composte da pochissimi membri, i quali osservavano una rigida compartimentazione e la massima segretezza» ${ }^{28}$, in cui pare ebbero un ruolo niente affatto secondario personaggi del calibro di Roberto Dotti e Luigi Cavallo, legati direttamente a Edgardo Sogno. Parrebbe - il condizionale è d'obbligo - che del gruppo di Simioni si siano perse le tracce per qualche anno, fino a quando, all'inizio del 1974, con la mediazione del deputato comunista Alberto Malagugini - suocero di Duccio Berio - il gruppo "regolò" i suoi conti con la giustizia italiana, proprio quando le $\mathrm{Br}$ passarono all'“attacco al cuore dello Stato" con il sequestro Sossi. Nel 1976 Simioni e compagni si trasferirono a Parigi, dove fondarono la scuola Agorà, che nell'agosto del 1977 cambiò il nome in Hyperion. Fino a qui, dunque, nulla di particolarmente nuovo.

Cosa fu dunque questa fantomatica scuola di lingue Hyperion? Secondo De Prospo e Priore fu chiaramente una «centrale del terrorismo internazionale», come recita il sottotitolo del libro. $\mathrm{O}$, più precisamente, dalla seconda metà degli anni Settanta

\footnotetext{
i fondatori dell'Hyperion avrebbero svolto in Francia un ruolo di coordinamento tra varie formazioni eversive europee e l'OLP. Il loro progetto sarebbe stato funzionale alla creazione sul suolo francese, di un foro delle forze eversive che avrebbe deciso il livello della tensione e dello scontro in Europa. Foro in cui più servizi segreti si sarebbero infiltrati non solo per acquisire notizie e informazioni, ma anche per manovrare i vari terrorismi e giocare partite a proprio favore, allo scopo di trarre
}

${ }^{28}$ DE PROSPO Silvano., PRIORE Rosario, op. cit., p. 89. 
vantaggi dalla conseguente destabilizzazione e dalle aumentate conflittualità interne dei governi limitrofi. ${ }^{29}$

Basandosi unicamente sulle rivelazioni di alcuni testimoni ed ex membri fuoriusciti (Antonio Savasta, Michele Galati, Roberto Buzzatti, Carlo Fioroni, Carlo Brogi, Gino Aldi) e su una serie di inchieste su di un traffico di armi fra l'OLP e le Br, gli autori ipotizzano che questa «struttura di coordinamento superiore, funzionale al collegamento tra le varie formazioni eversive italiane ed europee, alla formazione di quadri, al supporto alle latitanze e al lancio di campagne di propaganda» sia stata progettata e abbia iniziato a prendere forma già nel 1969 con i piani di Giangiacomo Feltrinelli e poi con quelli di Toni Negri. Una struttura che «ha fissato livelli, direzione, tempi della lotta armata europea e non solo [...] in vista di un unico processo di destabilizzazione dell'Alleanza atlantica», anche grazie al concorso, l'appoggio e la protezione dei servizi segreti francesi nell'ottica del «ritorno a una politica estera di supremazia» francese ${ }^{30}$. Una struttura con cui sarebbe poi entrato in contatto Moretti dopo la fine del sequestro Moro, per l'approvvigionamento di armi attraverso i palestinesi e per una gestione politica di più ampio respiro che legasse le $\mathrm{Br}$ alla lotta antimperialista mondiale a fianco degli altri gruppi terroristi europei (RAF, ETA, IRA, Action Directe). Dopo l'arresto di Moretti nel 1981, le Br sarebbero state capaci di rientrare in contatto con tale struttura tramite Riccardo Dura e Fulvia Miglietta, che permisero a Giovanni Senzani di partecipare a tre incontri a Parigi prima del suo arresto nel gennaio del 1982, momento nel quale la struttura scomparve poco a poco, anche a causa dell'arresto di due uomini chiave, secondo la ricostruzione degli autori, Vanni Mulinaris nel febbraio del 1982 e Jean-Louis Baudet ad inizio 1984.

Per quanto faccia un po' di luce sulla vicenda del cosiddetto Superclan - su cui poco si sa, anche per i molti silenzi delle istituzioni d'Oltralpe - la ricostruzione proposta da De Prospo e Priore ci sembra inficiata da un eccessivo dietrologismo, che porta gli autori a leggere gli eventi nell'ottica di un gioco manovrato da potenze straniere all'interno dei fragili equilibri della Guerra fredda e ad affermare che i gruppi extra

29 DE PROSPO Silvano, PRIORE Rosario, op. cit., p. 24.

3o DE PROSPO Silvano, PRIORE Rosario, op. cit., pp. 228, 255-256, 257. Parecchie pagine (pp. 163-202) sono dedicate alla "protezione francese" dell'Hyperion, soprattutto mediante l'attivismo dell'Abbé Pierre - la cui nipote, Françoise Marie Tüscher, era uno dei membri del Superclan -, il Comitato Vanni Mulinaris e la dottrina Mitterand, e ai gruppi dell'estrema sinistra francese degli anni Settanta, dalla Gauche proletariénne e i Matériaux pour l'intervention al CRISE, i NAPAP e Action Directe. 
parlamentari di sinistra non erano altro che volontarie o involontarie pedine di questo gioco3i. Un leitmotiv che ha riempito gli scaffali delle librerie negli ultimi vent'anni.

Ci sembra, invece, che sia Aldo Giannuli a mettere in luce in una maniera più equilibrata e debitrice del rigore storico il ruolo dei servizi segreti nella recente storia italiana (ed europea), seguendo la pionieristica intuizione di Franco De Felice sul "doppio Stato":

I servizi segreti, più o meno ufficiali o coperti, pubblici o privati, partecipano al pari di tutti gli altri soggetti alla lotta politica, sociale ed economica, fanno i loro giochi che a volte riescono e a volte falliscono, ma che sempre si intrecciano con le azioni degli altri, producendo, nella maggior parte dei casi, effetti imprevisti e non cercati da nessun attore. Certo i servizi segreti agiscono sulla lotta politica, ma ne sono anche agiti, sono costretti a modificare le loro strategie, a incassare insuccessi, a cercare alleanze impreviste, e qualche volta ne sono sconfitti al punto da esserne distrutti. $3^{2}$

${ }^{31}$ Politicamente errato e storiograficamente fuorviante ci sembra anche considerare tutti i gruppi della sinistra extra parlamentare italiana come partecipi delle azioni terroriste, mettendo nello stesso calderone Potere operaio, Lotta continua, Autonomia operaia, le Brigate rosse, Prima linea, ecc. Senza parlare del giudizio dato di Toni Negri, che viene ad essere quasi una sorta di Grande Vecchio in versione minore. Il tutto senza condannare lo scempio democratico del 7 aprile 1979 - quando il sostituto procuratore di Padova, Pietro Calogero, autorizzò l'arresto di oltre diecimila dirigenti e militanti di Autonomia operaia secondo l'ipotesi che fossero il cervello di un azione insurrezionale contro i poteri dello Stato - uno scempio che per gli autori tale non è stato. Una visione che purtroppo è diventata una delle vulgate più gettonate.

$3^{2}$ GIANNULI, Aldo, Il Noto servizio, cit., p. 19-20. Per quanto riguarda la questione del "doppio Stato", vedasi GIANNULI, Aldo, «Ma la tesi del doppio Stato non è una barzelletta», in Liberazione, 4 giugno 2009 [consultabile anche in www.aldogiannuli.it]. L'articolo di Giannuli è una risposta al dibattito scatenatosi in seguito al discorso del Presidente della Repubblica Giorgio Napolitano del 9 maggio 2009 riguardo alla validità della categoria del "doppio Stato", segnato dalle prese di posizione di Pier Luigi Battista, Paolo Persichetti, Marco Clementi, Vladimiro Satta, Giovanni Sabbatucci e Giuseppe De Lutiis, tra gli altri. Il riferimento all'intuizione defeliciana è contenuto in DE FELICE, Franco, «Doppia lealtà e doppio Stato», Studi Storici, 3/1989, pp. 493-563. Al riguardo vedasi anche gli interessanti interventi di SANTOMASSIMO, Gianpasquale «Il lungo dopoguerra repubblicano» e di SABBATUCCI, Giovanni, «Il "doppio Stato"» contenuti in Dimensioni e problemi della ricerca storica, 1/2009, rispettivamente alle pagine 62-70 e 89-93. Gli interventi sono il frutto del convegno «Franco De Felice storico e maestro» tenutosi all'Università di Roma la Sapienza il 9 ottobre 2008. 


\section{* L'autore}

Laureatosi presso l’Università degli Studi di Bologna con una tesi sul primo dopoguerra italiano, Steven Forti (Trento, 1981) è dottore di ricerca per l'Universidad Autónoma de Barcelona con una tesi centrata sulla questione del transito di dirigenti politici di sinistra al fascismo nell'Europa interbellica intitolata El peso de la nación. Nicola Bombacci, Paul Marion y Óscar Pérez Solís en la Europa de entreguerras. Le sue ricerche si focalizzano sulla storia politica e del pensiero politico nel XX secolo, con particolare attenzione allo studio biografico ed all'analisi del linguaggio politico. Collaboratore di varie riviste di storia contemporanea in Italia e Spagna (Memoria e Ricerca, Spagna Contemporanea, Storicamente, Nous Horitzons, Atlántica XXII), è stato uno dei fondatori di PRAXIS (Asociación de Jóvenes Investigadores en Historia y Ciencias Sociales) ed attualmente è membro del CEFID (Centre d'Estudis sobre les Epoques Franquista i Democràtica). Tra le sue ultime pubblicazioni ricordiamo «Otra deriva fascista. Paul Marion en la Francia de entreguerras» in GALLEGO, Ferran, MORENTE, Francisco (eds.), Rebeldes y reaccionarios. Intelectuales, fascismo y derecha radical en Europa, 1914-1956, Barcellona, El Viejo Topo, 2011.

URL: < http://studistorici.com/progett/autori/\#Forti >

\section{Per citare questo articolo:}

FORTI, Steven, «Aida. Le Italie segrete dalla Resistenza a Tangentopoli», Diacronie. Studi di Storia Contemporanea: Quando la classe operaia andava in paradiso, 13/2/2012,

URL:< http://www.studistorici.com/2012/02/13/forti_numero_9/ >

Diacronie Studi di Storia Contemporanea $\beta$ www.diacronie.it

Risorsa digitale indipendente a carattere storiografico. Uscita trimestrale. redazione.diacronie@hotmail.it

Comitato di redazione: Marco Abram - Giampaolo Amodei - Jacopo Bassi - Luca Bufarale - Alessandro Cattunar - Alice De Rensis Barbara Galimberti - Deborah Paci - Fausto Pietrancosta - Martina Sanna - Matteo Tomasoni - Luca Zuccolo

Diritti: gli articoli di Diacronie. Studi di Storia Contemporanea sono pubblicati sotto licenza Creative Commons 2.5 Possono essere riprodotti a patto di non modificarne i contenuti e di non usarli per fini commerciali. La citazione di estratti è comunque sempre autorizzata, nei limiti previsti dalla legge. 